\title{
GEDLIB: A C++ Library for Graph Edit Distance Computation
}

\author{
David B. Blumenthal ${ }^{1[0000-0001-8651-750 X]}$, Sébastien \\ Bougleux $^{2[0000-0002-4581-7570]}$, Johann Gamper ${ }^{10000-0002-7128-507 X]}$, and Luc \\ Brun $^{2}[0000-0002-1658-0527]$ \\ 1 Faculty of Computer Science, Free University of Bozen-Bolzano, Bolzano, Italy \\ 2 Normandie Univ, UNICAEN, ENSICAEN, CNRS, GREYC, Caen, France
}

Presented at the 12th IAPR TC15 Workshop on Graph-Based Representation in Pattern Recognition (GbR), 2019, June 19-21, Tours, France

(https://gbr2019.sciencesconf.org/)

\begin{abstract}
The graph edit distance (GED) is a flexible graph dissimilarity measure widely used within the structural pattern recognition field. In this paper, we present GEDLIB, a $\mathrm{C}++$ library for exactly or approximately computing GED. Many existing algorithms for GED are already implemented in GEDLIB. Moreover, GEDLIB is designed to be easily extensible: for implementing new edit cost functions and GED algorithms, it suffices to implement abstract classes contained in the library. For implementing these extensions, the user has access to a wide range of utilities, such as deep neural networks, support vector machines, mixed integer linear programming solvers, a blackbox optimizer, and solvers for the linear sum assignment problem with and without error-correction.
\end{abstract}

Keywords: Graph Edit Distance · Open Source Library · C++.

\section{Introduction}

Because of their expressiveness and versatility, labeled graphs are widely used to model various kinds of objects such as molecules, street networks, and images. Many pattern recognition problems defined over these domains presuppose the availability of a (dis-)similarity measure for labeled graphs. Despite the fact that its exact computation is $\mathcal{N} \mathcal{P}$-hard [31], one of the most widely used measures is the graph edit distance (GED). Given two labeled graphs $G$ and $H$, it is defined as $\operatorname{GED}(G, H):=\min _{P \in \Psi(G, H)} c(P)$, where $\Psi$ is the set of all edit paths between $G$ and $H$ and $c(P)$ denotes the cost of an edit path $P$. An edit path is a sequence of edit operations that transforms $G$ into $H$. There are six edit operations: substituting a node or an edge in $G$ by a node or an edge in $H$, deleting an edge or an isolated node from $G$, and inserting an edge or an isolated node into $H$. Each edit operation comes with an associated non-negative edit cost defined in terms of the node or edge labels involved in the operation; and the cost of an edit path is defined as the sum over the costs of its edit operations.

Over the past years, some exact and a lot of approximate algorithms for computing GED have been suggested. As the hardness of GED does not allow 
for a theoretical evaluation of approximate algorithms (the existence of any $\alpha$ approximation algorithm for GED would imply that the graph isomorphism problem, a prime candidate for an $\mathcal{N} \mathcal{P}$-intermediate problem, is in $\mathcal{P}$ ), these algorithms are typically evaluated empirically. In order for such a comparison to be fair, it is highly desirable that the compared algorithms be implemented within the same environment. However, to the best of our knowledge, no software is available that can be used for this purpose.

In this paper, we present the $\mathrm{C}++$ template library GEDLIB which is intended to fill this gap. GEDLIB is available on GitHub:

$$
\text { https://github.com/dbblumenthal/gedlib }
$$

In its current version, GEDLIB contains implementations of 24 different GED algorithms and 9 different edit cost functions. Further algorithms and edit costs can be implemented easily by implementing abstract classes contained in GEDLIB. For this, the user has access to standard libraries for blackbox optimization, mixed integer linear programming, the linear sum assignment problem with and without error-correction, deep neural networks, and support vector machines. GEDLIB provides a parser to load graphs given in the GXL file format. Alternatively, graphs with user-specified node ID, node, and edge label types can be constructed from within GEDLIB. Internally, GEDLIB uses the Boost Graph Library [22] for representing the graphs and Eigen [19] for matrix operations.

The remainder of this paper is organized as follows: In Section 2, the overall architecture of GEDLIB is sketched. In Section 3, the user interface is presented. In Section 4 and Section 5, the abstract classes for implementing GED algorithms and edit cost functions are described. Section 6 concludes the paper. Details, examples, and installation instructions can be found in the documentation.

\section{Overall Architecture}

Figure 1 shows the overall architecture of GEDLIB in a UML diagram. The entire library is contained in the namespace ged. The template parameters UserNodeID, UserNodeLabel, and UserEdgeLabel correspond to the types of the node IDs, the node labels, and the edge labels of the graphs provided by the user.

- The class template ged: :GEDEnv provides the user interface. Via its public member functions, graphs can be constructed or loaded from GXL files, edit costs can be set, the algorithms implemented in GEDLIB can be run, and the results of the runs can be obtained. For users who do not want to provide extensions for GEDLIB, it suffices to get familiar with this class template.

- The abstract class template ged::GEDMethod provides a generic interface for implementing algorithms that exactly or approximately compute GED.

- The abstract class templates ged: :LSBasedMethod, ged: :MIPBasedMethod, and ged::LSAPEBasedMethod are derived from the generic interface provided by ged: :GEDMethod. They yield more specialized interfaces for implementing methods using local search, mixed integer linear programming, and transformations to the linear sum assignment problem with error-correction. 


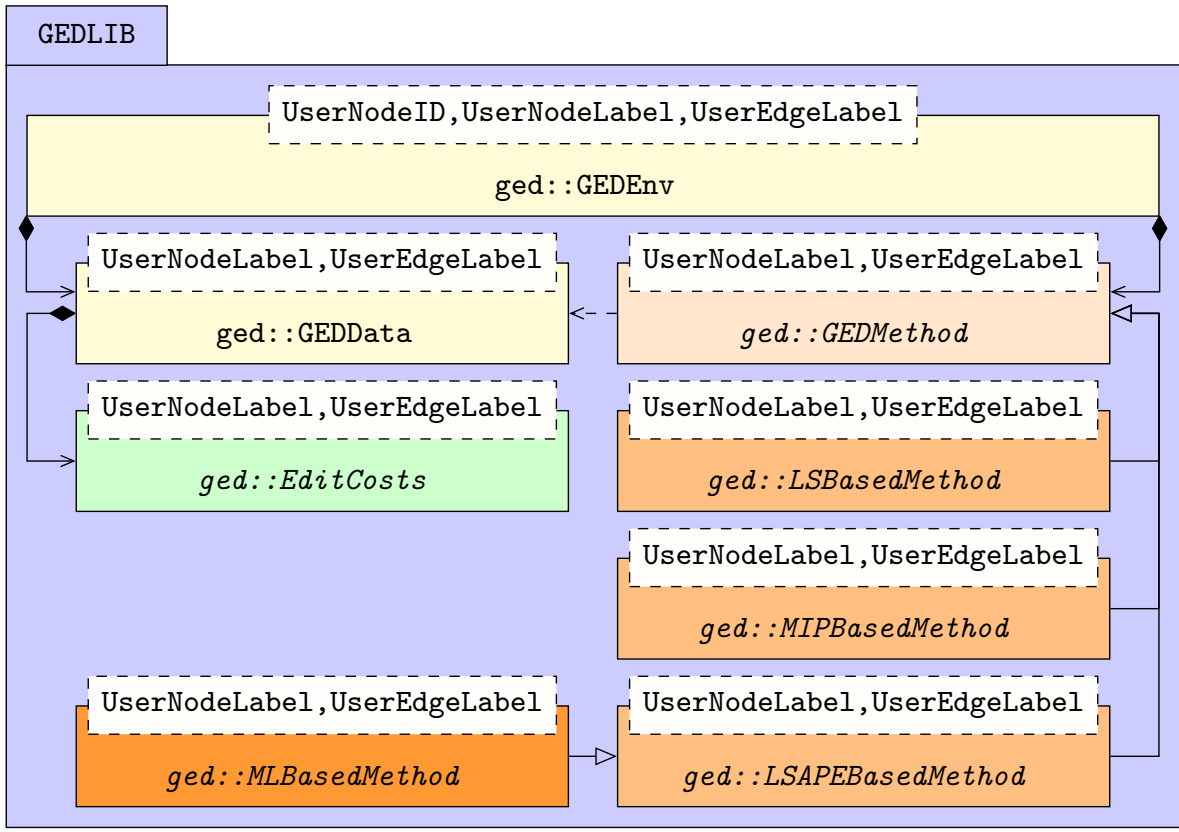

Fig. 1. The overall architecture of GEDLIB shown in a UML class diagram.

- The abstract class template ged: :MLBasedMethod is derived from the interface ged: :LSAPEBasedMethod. It can be used to implement algorithms that use deep neural networks or support vector machines for transforming GED to the linear sum assignment problem with error-correction.

- The class template ged: :GEDData contains the normalized input data on which all GED algorithms contained in GEDLIB operate. Via the public member functions of ged: :GEDData, derived classes of ged: :GEDMethod have access to the graphs that have been added to the environment and to the edit cost functions selected by the user.

- The abstract class template ged: EditCosts provides a generic interface for implementing edit cost functions.

\section{User Interface}

In Figure 2, the class template ged: : GEDEnv, which constitutes the user interface of GEDLIB, is displayed in detail. By calling add_graph(), add_node(), and add_edge (), the user can add labeled graphs to the environment. Alternatively, load_gxl_graphs () can be used to load graphs given in the GXL file format. For this, the template parameter UserNodeID must be set to ged: : GXLNodeID a. k. a. std: : string, and the template parameters UserNodeLabel and UserEdgeLabel must be set to ged: : GXLLabel a.k. a. std: :map<std: :string, std: :string>. 
Calls to set_edit_costs() add edit cost functions to the environment. The user can either select one of the predefined edit cost functions or use her own implementation of ged: :EditCosts. Calls to init() initialize the environment eagerly or lazily. If eager initialization is chosen, all edit costs between graphs contained in the environment are precomputed. Otherwise, the edit cost functions are evaluated on the fly. The member function set method() selects one of the GED algorithms available in GEDLIB. Some algorithms accept options, which can be passed to set method() as a string of the form "[--<option> $<\arg >$ ] [...]". Calls to init_method() initialize the selected method for runs between graphs contained in the environment, and calls to run method () run the method between two specified graphs. The results of the runs (lower and upper bounds, runtimes, etc.) can be accessed via various getter member function.

\begin{tabular}{|c|}
\hline $\begin{array}{l}\text { ged: : GEDEnv } \\
\end{array}$ \\
\hline // misc. variables \\
\hline $\begin{array}{ll}\text { + add_graph() } & / / \text { adds a graph to the environment } \\
\text { + add_node() } & / / \text { adds a node to a previously added graph } \\
\text { + add_edge() } & / / \text { adds an edge to a previously added graph } \\
\text { + load_gxl_graphs() } & / / \text { loads graphs given as GXL files } \\
\text { + set_edit_costs() } & / / \text { selects the edit costs } \\
\text { + init() } & / / \text { initializes the environment } \\
+ \text { set_method() } & / / \text { selects the GED method } \\
+ \text { init_method() } & / / \text { initializes the selected GED method } \\
+ \text { run_method() } & / / \text { runs the selected GED method } \\
\text {.. } & / / \text { misc. } \text { member functions }\end{array}$ \\
\hline
\end{tabular}

Fig. 2. The user interface ged: :GEDEnv.

\section{Abstract Classes for Implementing GED Algorithms}

Generic Interface. Figure 3 details the abstract class template ged: :GEDMethod, which provides the generic interface for implementing GED. The interface is defined by the virtual member functions starting with the prefix ged_. We here describe only the most important virtual member functions; the remaining ones are detailed in the documentation: ged_run_() runs the method between two input graphs, ged_init_ () initializes the methods for the graphs that have been added to the environment, and ged_parse_option_() parses the options of the method. The following existing algorithms already implemented in GEDLIB are directly derived classes of ged::GEDMethod: ged::BranchTight [2], ged::HED [17], ged: :Partition [32], ged: :Hybrid [32], ged: :SimulatedAnnealing [30], ged: : BranchCompact [32], ged: :AnchorAwareGED [14]. 


\begin{tabular}{|c|}
\hline 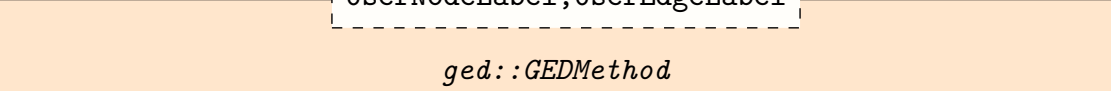 \\
\hline // misc. variables \\
\hline $\begin{array}{ll}\text { - ged_run_() } & / / \text { runs the method between two graphs } \\
\text { - ged_init_() } & / / \text { initializes the method for the graphs in ged_data } \\
\text { - ged_parse_option-() } / / \text { parses the options } \\
\text {... }\end{array}$ \\
\hline
\end{tabular}

Fig. 3. The generic interface ged: :GEDMethod.

Interface for Methods Based on the Linear Sum Assignment Problem with ErrorCorrection. A popular approach for approximating GED is to use transformations to the linear sum assignment problem with error-correction (LSAPE). An instance of LSAPE consists of a cost matrix $\mathbf{C}=\left(c_{i, k}\right) \in \mathbb{R}_{>0}^{(n+1) \times(m+1)}$. The task is to compute a mapping $\pi$ from rows to columns, such that each row except for $n+1$ and each column expect for $m+1$ is covered exactly once and $\mathbf{C}(\pi):=\sum_{(i, k) \in \pi} c_{i, k}$ is minimized. LSAPE can be solved optimally in cubic time [10]; in GEDLIB, we use the LSAPE toolbox [8] for solving LSAPE.

If LSAPE is used for approximating $\operatorname{GED}(G, H), n$ and $m$ are set to $\left|V^{G}\right|$ and $\left|V^{H}\right|$, the first $\left|V^{G}\right|$ rows of $\mathbf{C}$ are associated with the nodes of $G$, the first $\left|V^{H}\right|$ columns of $\mathbf{C}$ are associated with the nodes of $H$, and the last rows and columns are associated with dummy nodes used for codifying node insertions and deletions. With this setup, each LSAPE solution $\pi$ corresponds to a node map between $G$ and $H$, which, in turn, induces an edit path and hence an upper bound for $\operatorname{GED}(G, H)[6]$. LSAPE based heuristics for GED try to achieve tight upper bounds by encoding structural information of the input graphs into C. Moreover, some of them construct $\mathbf{C}$ such that $\min _{\pi} \mathbf{C}(\pi)$ lower bounds GED.

Figure 4 shows the abstract class template ged: :LSAPEBasedMethod, which provides the interface for implementing heuristics of this kind. The interface is defined by the virtual member functions starting with the prefix $l$ sape. The most important one is lsape_populate_instance_( ), which populates the LSAPE instance $\mathbf{C}$. The following algorithms implemented in GEDLIB are directly derived classes of ged::LSAPEBasedMethod: ged::Bipartite [26], ged::Branch [2], ged::BranchFast [2], ged::Node [21], ged::BranchUniform [32], ged::Ring [3], ged::Subgraph [12], ged::Walks [18]. Additionally, all derived classes of ged::LSAPEBasedMethod can be run with the node centralities suggested in [27].

Interface for Methods Based on Machine Learning. Recently, it has been suggested to use deep neural networks or support vector machines for carrying out the transformation from GED to LSAPE. Given two graphs $G$ and $H$, feature vectors are constructed for all node substitutions, deletions, and insertions, and the matrix $\mathbf{C}$ is defined as $c_{i, k}:=1-p^{\star}(i, k)$. Here, $p^{\star}(i, k)$ is the confidence of a machine learning framework (either a deep neural network or a support 


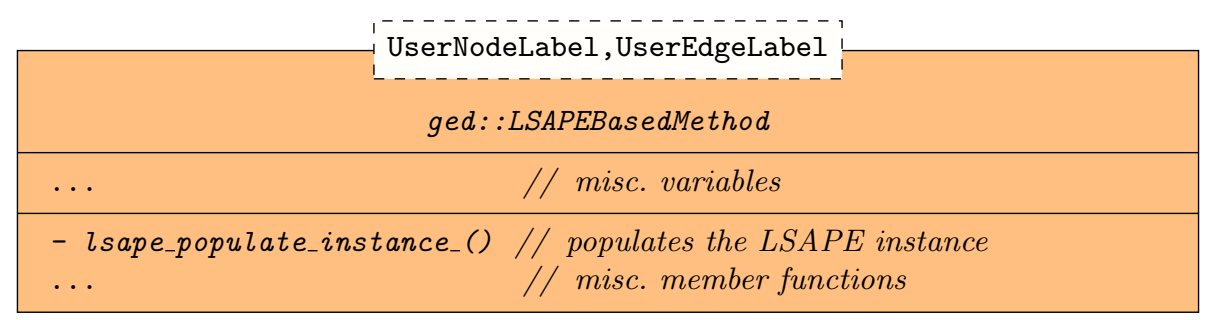

Fig. 4. The interface ged: :LSAPEBasedMethod for methods based on LSAPE.

vector machine) that the feature vector associated to the node edit operation corresponding to row $i$ and column $k$ is contained in an optimal node map.

Figure 5 details the abstract class template ged::MLBasedMethod, which provides the interface for algorithm adopting this paradigm. For implementing the interface, it suffices to override the virtual member functions starting with the prefix $m l_{-}$. The most important ones are the three virtual member functions of the form $m l_{-}$populate_*feature_vector_( $)$, which construct the feature vectors associated to the node edit operations. Derived classes of ged::MLBasedMethod do not have to implement the machine learning frameworks, as ged::MLBasedMethod offers support for artificial deep neural networks (using FANN [24]) and support vector machines (using LIBSVM [13]). The following algorithms implemented in GEDLIB are directly derived classes of ged: :MLBasedMethod: ged: :BipartiteML [28], ged: :RingML [4].

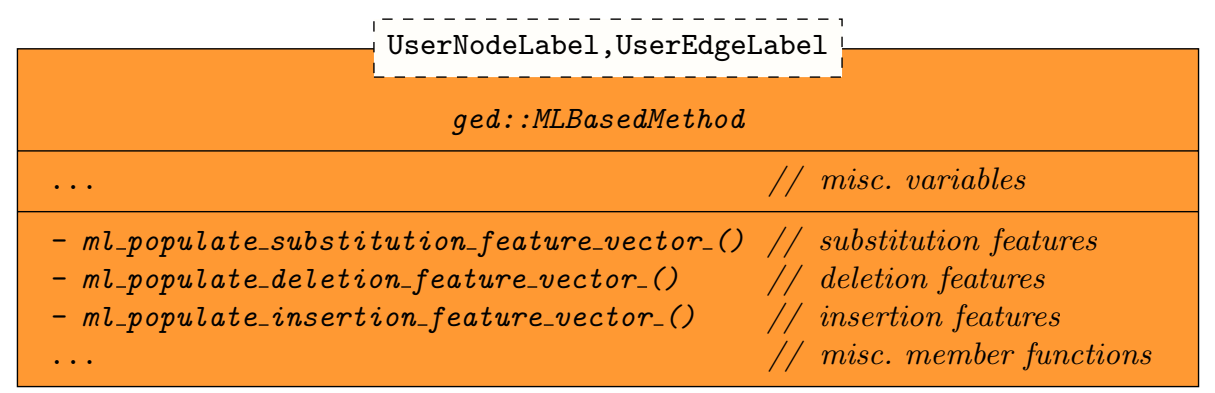

Fig. 5. The interface ged: :MLBasedMethod for LSAPE based methods that use machine learning techniques for populating their LSAPE instances.

Interface for Methods Based on Mixed Integer Programming. Another approach for exactly or approximately computing GED is to rephrase the problem of computing $\operatorname{GED}(G, H)$ as a mixed integer programming (MIP) problem. $\operatorname{GED}(G, H)$ can then be computed exactly by calling an MIP solver. Alternatively, lower bounds for $\operatorname{GED}(G, H)$ can be obtained by solving the linear programming (LP) relaxations of the MIP formulations. 
Figure 6 shows the abstract class template ged::MIPBasedMethod, which provides the interface for GED algorithms that use MIP formulations. The virtual member functions that define the interface start with the prefix mi $p_{-}$. The most important one is mip_populate_mode $z_{-}()$, which constructs the employed MIP formulation and must be overridden by all derived classes. In GEDLIB, we use Gurobi [20] as our MIP and LP solver. Gurobi is commercial software but offers a free academic license. For users who cannot obtain a license for Gurobi, the installation script distributed with GEDLIB offers the option to install GEDLIB without ged: :MIPBasedMethod and its derived classes. The following algorithms implemented in GEDLIB are directly derived classes of ged: :MIPBasedMethod: ged: :F1 [23], ged: :F2 [23], ged: :CompactMIP [6], ged: :BLPNoEdgeLabels [21].

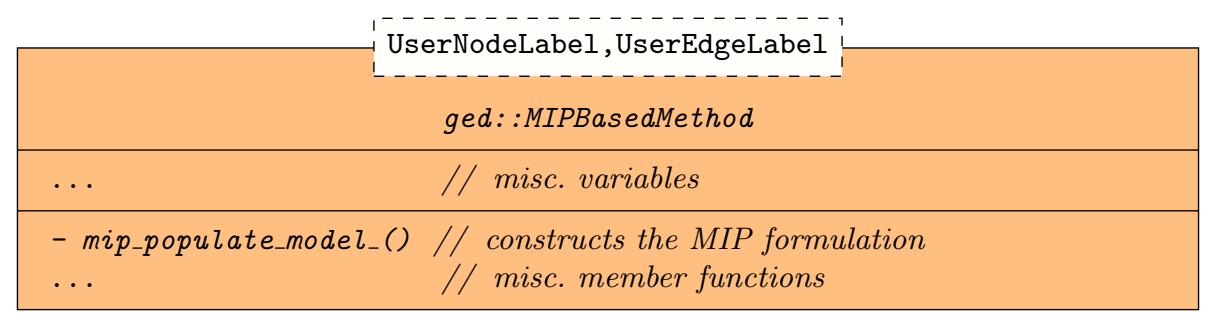

Fig. 6. The interface ged: :MIPBasedMethod for methods based on MIP.

Interface for Methods Based on Local Search. Another popular approach for upper bounding GED is to use variants of local search to systematically vary a previously computed or randomly generated node map, such that the cost of the induced edit path decreases. Figure 7 shows the abstract class template ged: :LSBasedMethod, which provides the interface for algorithms using local search. The prefix $l s_{-}$marks the virtual member functions defining the interface. The most important one is $l_{s_{-}} r u n_{-} f r o m_{-} i n i t i a l_{-} s o l u t i o n_{-}()$, which runs the local search from an initial node map. The following algorithms implemented in GEDLIB are directly derived classes of ged: :LSBasedMethod: ged: : IPFP [5, 9,11], ged: :BPBeam [16,29], ged: :Refine [31]. Moreover, ged: :LSBasedMethod provides support for running all derived classes with parallel multi-start as suggested in [15], and stochastic generators as suggested in [7].

\section{Abstract Class for Implementing Edit Costs}

Figure 8 shows the abstract class template ged: :EditCosts, which provided the interface for implementing edit cost functions. The virtual member functions *_de $\imath_{-}$cost_fun() compute the cost of deleting a node or an edge with a given label, the functions $*_{-} i n s_{-} \cos t_{-} f u n()$ compute the insertions costs, and the functions *_rel_cost_fun() compute the costs for relabeling a node or an 


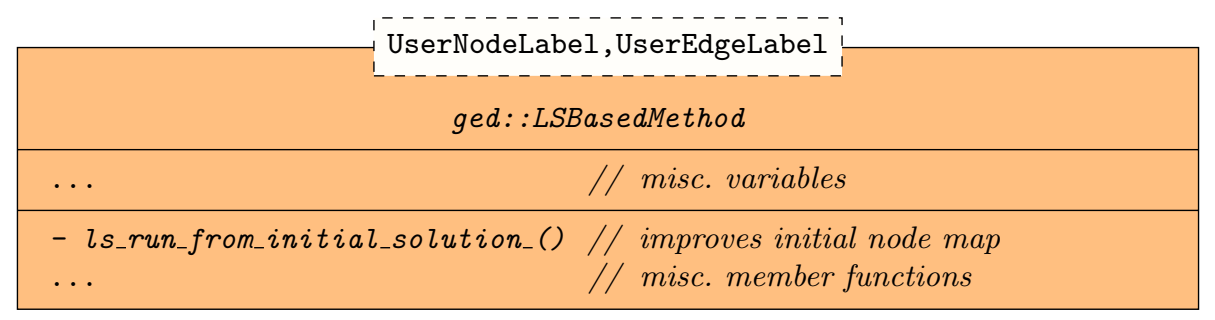

Fig. 7. The interface ged: :LSBasedMethod for methods based on local search.

edge. The functions vectorize_*_label() return vector representations of the node and the edge labels, which are required by some methods. In GEDLIB, edit costs are available for the datasets AIDS, FINGERPRINT, GREC, LETTER, MUTAGENICITY, and PROTEIN from the IAM Graph Database [25], for the datasets ACYCLIC, ALKANE, PAH, and MAO from GREYC's Chemistry Dataset (available at https://brunl01.users.greyc.fr/CHEMISTRY/), and for the dataset CMU-GED from the Graph Data Repository for Graph Edit Distance [1]. We also provide constant edit cost functions that can be used with any data.

\begin{tabular}{|c|}
\hline ged: :EditCosts \\
\hline 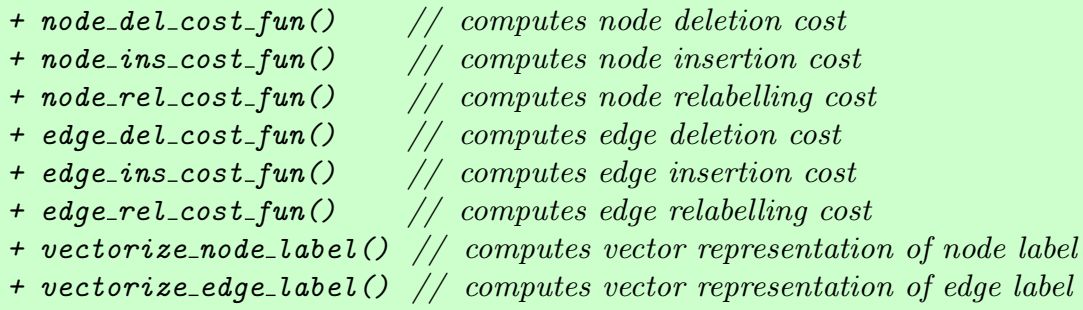 \\
\hline
\end{tabular}

Fig. 8. The interface ged: :EditCosts for implementing edit costs.

\section{Conclusions and Future Work}

In this paper, we have presented GEDLIB, a C++ library for GED computations. GEDLIB currently implements 24 different GED algorithms and 9 different edit cost functions designed for datasets which are widely used in the research community. In the future, we will provide Python and MATLAB bindings for better usability. Moreover, we would like to encourage authors of algorithms and edit costs that are not implemented in GEDLIB to commit their work to GEDLIB. 


\section{References}

1. Abu-Aisheh, Z., Raveaux, R., Ramel, J.: A graph database repository and performance evaluation metrics for graph edit distance. In: GbRPR 2015. LNCS, vol. 9069, pp. 138-147 (2015). https://doi.org/10.1007/978-3-319-18224-7_14, http://www.rfai.li.univ-tours.fr/PublicData/GDR4GED/home.html

2. Blumenthal, D.B., Gamper, J.: Improved lower bounds for graph edit distance. IEEE Trans. Knowl. Data Eng. 30(3), 503-516 (2018). https://doi.org/10.1109/TKDE.2017.2772243

3. Blumenthal, D.B., Bougleux, S., Gamper, J., Brun, L.: Ring based approximation of graph edit distance. In: S+SSPR 2018. LNCS, vol. 11004, pp. 293-303 (2018). https://doi.org/10.1007/978-3-319-97785-0_28

4. Blumenthal, D.B., Bougleux, S., Gamper, J., Brun, L.: Upper bounding GED via transformations to LSAPE based on rings and machine learning (2018), submitted

5. Blumenthal, D.B., Daller, E., Bougleux, S., Brun, L., Gamper, J.: Quasimetric graph edit distance as a compact quadratic assignment problem. In: ICPR 2018. pp. 934-939 (2018)

6. Blumenthal, D.B., Gamper, J.: On the exact computation of the graph edit distance. Pattern Recognit. Lett. (2018). https://doi.org/10.1016/j.patrec.2018.05.002

7. Boria, N., Bougleux, S., Brun, L.: Approximating GED using a stochastic generator and multistart IPFP. In: S+SSPR 2018. pp. 460-469 (2018). https://doi.org/10.1007/978-3-319-97785-0_44

8. Bougleux, S., Brun, L.: Linear sum assignment with edition. arXiv 1603.04380 [cs.DS] (2016), https://bougleux.users.greyc.fr/lsape/

9. Bougleux, S., Brun, L., Carletti, V., Foggia, P., Gaüzère, B., Vento, M.: Graph edit distance as a quadratic assignment problem. Pattern Recognit. Lett. 87, 3846 (2017). https://doi.org/10.1016/j.patrec.2016.10.001

10. Bougleux, S., Gaüzère, B., Blumenthal, D.B., Brun, L.: Fast linear sum assignment with error-correction and no cost constraints. Pattern Recognit. Lett. (2018). https://doi.org/10.1016/j.patrec.2018.03.032

11. Bougleux, S., Gaüzère, B., Brun, L.: Graph edit distance as a quadratic program. In: ICPR 2016. pp. 1701-1706 (2016). https://doi.org/10.1109/ICPR.2016.7899881

12. Carletti, V., Gaüzère, B., Brun, L., Vento, M.: Approximate graph edit distance computation combining bipartite matching and exact neighborhood substructure distance. In: GbRPR 2015. LNCS, vol. 9069, pp. 188-197 (2015). https://doi.org/10.1007/978-3-319-18224-7_19

13. Chang, C.C., Lin, C.J.: LIBSVM: A library for support vector machines. ACM Trans. Intell. Syst. Technol. 2(3), 27 (2011). https://doi.org/10.1145/1961189.1961199, https://www.csie.ntu.edu.tw/ $\sim$ cjlin/libsvm/

14. Chang, L., Feng, X., Lin, X., Qin, L., Zhang, W.: Efficient graph edit distance computation and verification via anchor-aware lower bound estimation. arXiv 1709.06810 [cs.DB] (2017)

15. Daller, É., Bougleux, S., Gaüzère, B., Brun, L.: Approximate graph edit distance by several local searches in parallel. In: ICPRAM 2018. pp. 149-158 (2018). https://doi.org/10.5220/0006599901490158

16. Ferrer, M., Serratosa, F., Riesen, K.: A first step towards exact graph edit distance using bipartite graph matching. In: GbRPR 2015. LNCS, vol. 9069, pp. 77-86 (2015). https://doi.org/10.1007/978-3-319-18224-7_8 
17. Fischer, A., Suen, C.Y., Frinken, V., Riesen, K., Bunke, H.: Approximation of graph edit distance based on Hausdorff matching. Pattern Recognit. 48(2), 331343 (2015). https://doi.org/10.1016/j.patcog.2014.07.015

18. Gauzère, B., Bougleux, S., Riesen, K., Brun, L.: Approximate graph edit distance guided by bipartite matching of bags of walks. In: S+SSPR 2014. LNCS, vol. 8621, pp. 73-82 (2014). https://doi.org/10.1007/978-3-662-44415-3_8

19. Guennebaud, G., Jacob, B., et al.: Eigen v3 (2010), http://eigen.tuxfamily.org

20. Gurobi Optimization, LLC: Gurobi optimizer reference manual (2018), http:// www. gurobi.com

21. Justice, D., Hero, A.: A binary linear programming formulation of the graph edit distance. IEEE Trans. Pattern Anal. Mach. Intell. 28(8), 1200-1214 (2006). https://doi.org/10.1109/TPAMI.2006.152

22. Lee, L., Lumsdaine, A., Siek, J.: The Boost Graph Library: User Guide and Reference Manual (2002), https://www.boost.org/doc/libs/1_68_0/libs/graph/ doc/index.html

23. Lerouge, J., Abu-Aisheh, Z., Raveaux, R., Héroux, P., Adam, S.: New binary linear programming formulation to compute the graph edit distance. Pattern Recognit. 72, 254-265 (2017). https://doi.org/10.1016/j.patcog.2017.07.029

24. Nissen, S.: Implementation of a fast artificial neural network library (FANN). Tech. rep., Department of Computer Science, University of Copenhagen (DIKU) (2003), http://leenissen.dk/fann/wp/

25. Riesen, K., Bunke, H.: IAM graph database repository for graph based pattern recognition and machine learning. In: S+SSPR 2008. LNCS, vol. 5342, pp. 287297 (2008). https://doi.org/10.1007/978-3-540-89689-0_33, http://www.fki.inf. unibe.ch/databases/iam-graph-database

26. Riesen, K., Bunke, H.: Approximate graph edit distance computation by means of bipartite graph matching. Image Vis. Comput. 27(7), 950-959 (2009). https://doi.org/10.1016/j.imavis.2008.04.004

27. Riesen, K., Bunke, H., Fischer, A.: Improving graph edit distance approximation by centrality measures. In: ICPR 2014. pp. 3910-3914 (2014). https://doi.org/10.1109/ICPR.2014.671

28. Riesen, K., Ferrer, M.: Predicting the correctness of node assignments in bipartite graph matching. Pattern Recognit. Lett. 69, 8-14 (2016). https://doi.org/10.1016/j.patrec.2015.10.007

29. Riesen, K., Fischer, A., Bunke, H.: Combining bipartite graph matching and beam search for graph edit distance approximation. In: ANNPR 2014. LNCS, vol. 8774, pp. 117-128 (2014). https://doi.org/10.1007/978-3-319-11656-3_11

30. Riesen, K., Fischer, A., Bunke, H.: Improved graph edit distance approximation with simulated annealing. In: GbRPR 2017. LNCS, vol. 10310, pp. 222-231 (2017). https://doi.org/10.1007/978-3-319-58961-9_20

31. Zeng, Z., Tung, A.K.H., Wang, J., Feng, J., Zhou, L.: Comparing stars: On approximating graph edit distance. PVLDB 2(1), 25-36 (2009). https://doi.org/10.14778/1687627.1687631

32. Zheng, W., Zou, L., Lian, X., Wang, D., Zhao, D.: Efficient graph similarity search over large graph databases. IEEE Trans. Knowl. Data Eng. 27(4), 964-978 (2015). https://doi.org/10.1109/TKDE.2014.2349924 